\title{
Perfil de los menores infractores extranjeros acompañados y no acompañados en Asturias
}

\author{
Profile of accompanied and unaccompanied foreign juvenile \\ delinquency in Asturias
}

\author{
Asunción Fernández-Suárez ${ }^{\mathrm{a}}$, Beatriz Pérez Sánchez ${ }^{\mathrm{b}}$, Laura Fernández-Alonso ${ }^{\mathrm{a}}$, Juan Herrero Olaizola ${ }^{\mathrm{a}}$ \\ y Francisco Javier Rodríguez-Díaz \\ ${ }^{a}$ Universidad de Oviedo, Principado de Asturias, España ${ }^{b}$ Universidad de La Frontera, Temuco, Chile
}

\begin{abstract}
Resumen: La alarma social generada por la delincuencia juvenil se ve incrementada cuando el infractor, además, es extranjero. El objetivo de este trabajo es analizar el perfil sociodemográfico, la problemática psicológica y delictiva de estos jóvenes, estableciendo una comparación entre los menores extranjeros no acompañados (MENAs) y los acompañados (procedentes de reagrupaciones familiares). La muestra se compone del total de menores infractores extranjeros en el Principado de Asturias (España) en $2012(N=90 ; n=61$ acompañados, $n=29$ no acompañados). Se utiliza el estadístico $X^{2} /$ la Prueba $U$ de Mann-Withney para establecer dicha comparación, presentando el Odds Ratio/el estadístico $d$ de Cohen como medida de la asociación/tamaño del efecto. Los resultados ofrecen similitudes entre ambos grupos en edad (15.5 años aproximadamente) y tipo de la primera infracción cometida (contra el patrimonio); sin embargo, ofrecen diferencias en base al género, la edad de llegada a España, el patrón de consumo de sustancias, la problemática psicológica, el nivel de reincidencia y las infracciones cometidas y medidas judiciales aplicadas a lo largo de sus carreras delictivas. Los resultados refieren que no se trata de un colectivo homogéneo, siendo discutidas sus implicaciones de cara a una orientación en la intervención desde el Sistema de Protección y Justicia Juvenil.
\end{abstract}

Palabras clave: menor infractor, extranjero, abuso de sustancias, reincidencia, intervención.
Abstract: The social alarm generated by juvenile delinquency is increased when the offender is also foreign. The aim of this paper is to analyze the socio-demographic, psychological and criminal problems of these children, establishing a comparison of unaccompanied foreign minors (MENAs, by its acronym in Spanish) and accompanied foreign minors (from family reunification). The sample consists of all offenders foreign minors in the Principality of Asturias (Spain) in 2012 ( $N=90 ; n=61$ accompanied; $n=29$ unaccompanied). $X^{2} / U$ test of Mann-Whitney statistical was used to set the comparison, presenting the Cohen's Odds Ratio/d as a measure of association/effect size. The results showed similarities between the two groups in age (15.5 years) and the type of first offense (against property). However, there are some differences by gender, age of arrival in Spain, the pattern of substance use, psychological problems, the level of recidivism, offenses and judicial measures applied throughout their criminal careers. The results refer which is not a homogeneous group, being discussed the implications to a counseling intervention from the Protection and Juvenile Justice System.

Keywords: minor offender, foreign, substance abuse, recidivism, intervention.

Investigación financiada por la Fundación para el Fomento en Asturias de la Investigación Científica Aplicada y la Tecnología (FICYT), mediante Becas Severo Ochoa (BP09147 y BP13134); y el Ministerio de Educación, Cultura y Deporte por ayudas para contratos predoctorales para Formación de Profesorado Universitario (FPU 13/04310). 
Contacto: A. Fernández-Suárez. Facultad de Psicología, Despacho 215. Plaza Feijoó s/n. C.P. 33003, Oviedo, Asturias, España. Correo electrónico: fernandezasuncion@uniovi.es

Cómo citar: Fernández-Suárez, A., Pérez Sánchez, B., Fernández-Alonso, L., Herrero Olaizola, J. y RodríguezDíaz, F. J. (2015). Perfil de los menores infractores extranjeros acompañados y no acompañados en Asturias. Revista de Psicología, 24(1), 1-18. http://dx.doi.org/10.5354/0719-0581.2015.36911 


\section{Introducción}

La inquietud social que generan los delitos cometidos por menores de edad es evidente incluso en la exposición de motivos de la última reforma de la Ley Orgánica 5/2000, de 12 de enero, reguladora de la responsabilidad penal de los menores en España (de aquí en adelante, LORPM) efectuada a través de la Ley Orgánica 8/2006, de 4 de diciembre. En la misma se plantea la existencia de una "gran preocupación social y un desgaste de la credibilidad de la ley por la sensación de impunidad de las infracciones más cotidianas y frecuentemente cometidas por estos menores" (Agencia Estatal Boletín Oficial del Estado, 2006, pp. 42700), argumentos que justifican el endurecimiento de las sanciones aplicadas a los menores infractores. Esta realidad se vuelve todavía más alarmante cuando el delincuente, además de ser menor, es extranjero; el rechazo hacia este colectivo es mucho más acusado. Así lo demuestra Moreno (2012), quien en su estudio acerca de la percepción y opinión de los menores extranjeros no acompañados (de aquí en adelante, MENAs) en el País Vasco, establece que la opción de la repatriación de los menores es la más apoyada por la población, independientemente de si dichos menores delinquen o no.

$\mathrm{Si}$ se analizan los principales modelos explicativos de la conducta delictiva, fundamentalmente desde el punto de vista de los factores de riesgo y protección para el inicio y mantenimiento de la conducta desadaptada, estos menores se convierten en un colectivo especialmente vulnerable (Musitu, Moreno y Murgui, 2007). La literatura científica señala como factores de riesgo para la delincuencia -cualquier elemento personal, familiar o social que, de acuerdo con la investigación, se asocia a un mayor riesgo delictivo (Redondo,
2008) - la inestabilidad/movilidad residencial (Paíno, 2008; Redondo, 2008; Rodríguez y Becedóniz, 2007), la percepción de ser objeto de prejuicio (Herrero, Rodríguez Díaz y Musitu, 2014; Rodríguez, Herrero, Ovejero y Torres, 2009; Valdenegro, 2005) y el apoyo social percibido (Estrada, Herrero y Rodríguez Díaz, 2014; Leiva, Pineda y Encina, 2013; Valdenegro, 2005), entre otros muchos, los cuales son elementos presentes en los menores extranjeros. Se trata de un colectivo con altas tasas no solo de movilidad residencial (debido al propio proceso migratorio, mucho más acentuado en el caso de los MENAs), sino también de cambios en el núcleo familiar (sobre todo en los casos de reagrupación familiar). Asimismo, el apoyo ejercido por la familia desaparece totalmente en el caso de los MENAs y de manera parcial en los menores acompañados, ya que aunque sí cuentan con la presencia de su familia más cercana (padres y hermanos), en la mayoría de los casos la familia extensa deja de ejercer el rol de apoyo característico.

De este modo, muchas de las explicaciones psicológicas de la delincuencia podrían ser aplicadas al colectivo de menores infractores extranjeros. La teoría general de la tensión (Garrido, Stangeland y Redondo, 2006) entiende que diversas fuentes de tensión (como el propio proceso migratorio) pueden generar en el sujeto emociones negativas que energizan una conducta correctora de dicha situación, como puede ser la conducta delictiva. Del mismo modo, según Valverde (2002), el proceso de inadaptación social se inicia con un conflicto anómico entre las expectativas sociales del menor y la carencia de medios para alcanzarlas, surgiendo la conducta antisocial como un camino alternativo para la adaptación a una nueva realidad (el país de destino). Por último, la teoría de los vínculos sociales (Hirschi, 
1969) postula la existencia de una serie de contextos principales que sirven de unión entre jóvenes y sociedad: la familia, la escuela, el grupo de amigos y las pautas de acción convencionales; de modo que la etiología de la conducta antisocial reside precisamente en la ruptura de los vínculos con estos contextos. En el caso de los menores extranjeros, todos ellos se rompen, exceptuando el vínculo con la familia nuclear en el caso de menores extranjeros acompañados.

Profundizar en la realidad de estos menores lleva a presentar las cifras de delincuencia juvenil asociadas concretamente a la población extranjera, tanto a nivel nacional como en el Principado de Asturias; no se puede olvidar que las limitaciones presentadas por las estadísticas oficiales a la hora de aportar la realidad de la criminalidad (cambios en la forma de recogida de la información, retrasos en sus publicaciones, ausencia de sistemas de información que aglutinen datos regionales y ofrezcan una visión nacional, etc.), provocan que exista una elevada cifra negra respecto de la delincuencia juvenil, en la medida en la que muchas infraccio- nes no llegan al conocimiento de las instancias oficiales (Montero, 2014). Sobre la base de las cifras recogidas en la figura 1 y aportadas por el Instituto Nacional de Estadística de España (2013) -de aquí en adelante, INE - respecto del número de menores condenados, se observa que mientras entre los nacionales se sucede un leve descenso a partir del año 2010, la población extranjera se estabiliza alrededor de los 3500 condenados, constituyendo en el año 2013 -último año del que se aportan estadísticas-el $23.95 \%$ de la población total de menores infractores.

Analizando las infracciones cometidas en el año 2013 por menores nacionales y extranjeros, se puede comprobar en la tabla 1 que estos últimos cometen prácticamente la mitad de cada tipo de infracción, exceptuando el homicidio y delitos contra el orden público -los porcentajes se reducen en torno al 38\%; ello, a pesar de que, como se ha visto en la figura 1, solo suponen el $24 \%$ del total de menores infractores. El análisis de las medidas judiciales impuestas ofrece una situación idéntica, tal como se puede comprobar en la tabla 2.

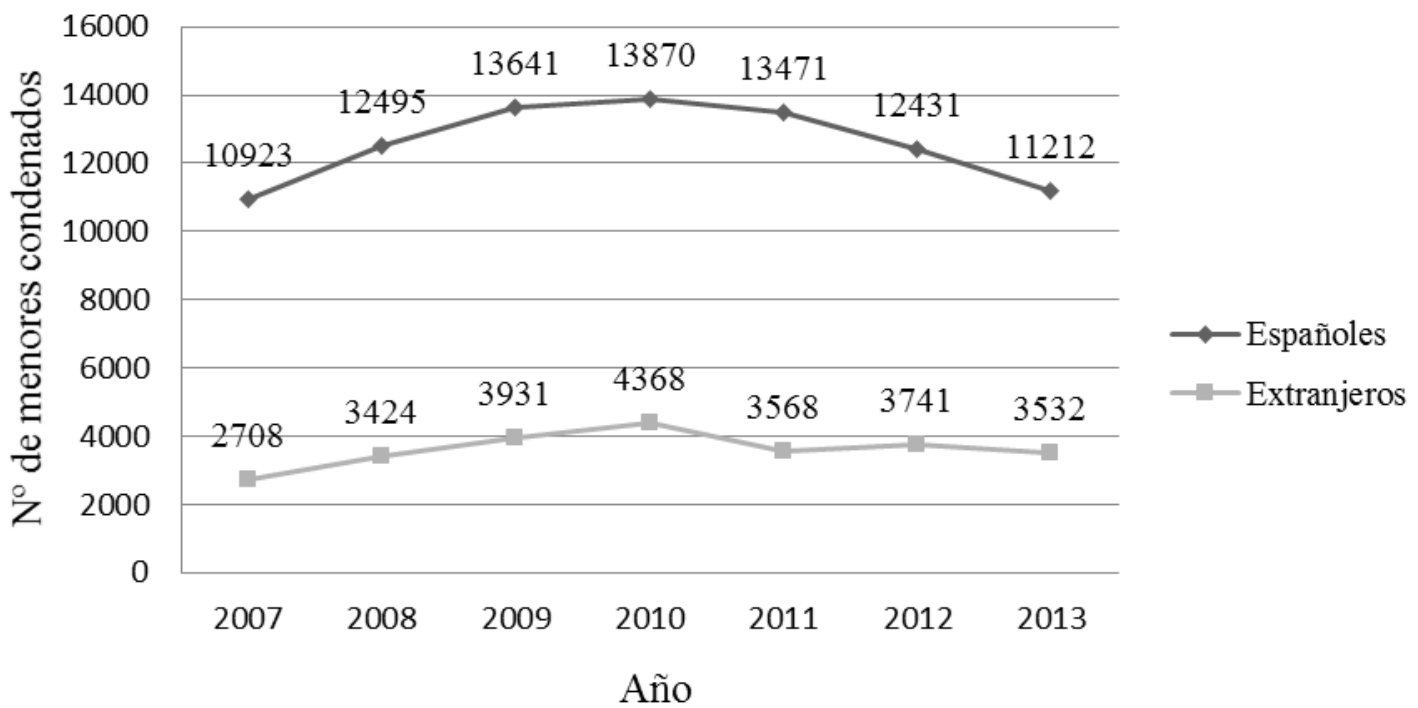

Figura 1. Estadística de menores condenados en España, según nacionalidad (datos aportados por el INE, 2013). 
Tabla 1

Estadísticas de infracciones cometidas por menores en 2013, según nacionalidad

\begin{tabular}{lrrc} 
& Españoles & Extranjeros & $\%$ Extranjeros \\
\hline Homicidio y sus formas & 49 & 30 & $38.0 \%$ \\
Lesiones & 2149 & 1671 & $43.7 \%$ \\
Contra la libertad & 797 & 601 & $43.0 \%$ \\
Torturas e integridad moral & 1502 & 1298 & $46.4 \%$ \\
Contra la libertad e indemnidad sexuales & 232 & 163 & $41.3 \%$ \\
Contra la intimidad, derecho a la propia imagen & 114 & 90 & $44.1 \%$ \\
Contra el patrimonio y el orden socioeconómico & 9198 & 6532 & $41.5 \%$ \\
Contra la seguridad colectiva & 1279 & 1086 & $45.9 \%$ \\
De las falsedades & 50 & 34 & $40.5 \%$ \\
Contra la Administración de Justicia & 581 & 465 & $44.5 \%$ \\
Contra el orden público & 676 & 426 & $38.7 \%$ \\
\hline
\end{tabular}

Nota: Datos aportados por el INE, 2013.

Tabla 2

Estadística de medidas judiciales impuestas a los menores en 2013, según nacionalidad

\begin{tabular}{lrrc} 
& Españoles & Extranjeros & $\%$ Extranjeros \\
\hline Asistencia a un centro de día & 130 & 111 & $46.1 \%$ \\
Amonestación & 756 & 616 & $44.9 \%$ \\
Convivencia con otra persona, familia o grupo & & & \\
educativo & 383 & 335 & $46.7 \%$ \\
Internamiento abierto & 153 & 119 & $43.8 \%$ \\
Internamiento cerrado & 611 & 333 & $35.3 \%$ \\
Internamiento semiabierto & 2945 & 1938 & $39.7 \%$ \\
Internamiento terapéutico & 455 & 365 & $44.5 \%$ \\
Libertad vigilada & 9413 & 6815 & $42.0 \%$ \\
Prohibición de aproximarse/comunicarse con la & & & \\
víctima & 673 & 543 & $44.7 \%$ \\
Prestación en beneficio comunidad & 4303 & 3298 & $43.4 \%$ \\
Permanencia de fin de semana & 1255 & 914 & $42.1 \%$ \\
Privación de permiso de conducir & 91 & 81 & $47.1 \%$ \\
Realización de tareas socioeducativas & 2289 & 1830 & $44.4 \%$ \\
Tratamiento ambulatorio & 314 & 255 & $44.8 \%$ \\
\hline
\end{tabular}

Nota: Datos aportados por el INE, 2013. 
La situación de los menores condenados españoles en el Principado de Asturias refleja un leve descenso a partir del año 2009 (exceptuando el repunte existente en el año 2012); mientras que los extranjeros van en aumento desde el año 2011, constituyendo en 2013 el $28.9 \%$, tasa ligeramente superior a la existente a nivel nacional, la cual rondaba el $24 \%$. Sin embargo, la población de menores en Asturias puede ayudar a explicar dichas cifras: mientras la población de españoles va en descenso, la de extranjeros permanece establece. No obstante, se comprueba una sobrerrepresentación de este colectivo en el Sistema de Justicia Juvenil de Asturias: en el año 2013 los extranjeros suponen el $11.3 \%$ de la población de menores en edad de responsabilidad penal (33620 españoles y 4231 extranjeros), siendo responsables del $28.9 \%$ de la delincuencia juvenil.

Esta realidad lleva a plantear estudios que comienzan a establecer perfiles diferenciados, sugiriendo que el colectivo de menores infractores no es un grupo homogéneo. El grupo más documentado a nivel científico es el de los MENAs. El Real Decreto $557 / 2011$, de 20 de abril, por el que se aprueba el Reglamento de la Ley Orgánica 4/2000, sobre derechos y libertades de los extranjeros en España y su integración social (reformado por la Ley Orgánica 2/2009) les define en su Art. 189, como aquellos "menores de dieciocho años que llegan a territorio español sin venir acompañados de un adulto responsable de ellos, ya sea legalmente o con arreglo a la costumbre, apreciándose riesgo de desprotección" (BOE, 2011, pp. 43956). Suponen un problema emergente, el cual apunta a la necesidad de ser gestionado por el Sistema de Protección de menores.

Los estudios señalan que la mayoría de los MENAs en España son varones (Bermejo, Cario, Dünkel, Patanè y Zapulla, 2006;
Capdevila y Ferrer, 2003; Markez y Pastor, 2009) y marroquíes (Bermejo et al., 2006; Capdevila y Ferrer, 2003; Markez y Pastor, 2009; Procuradora General del Principado de Asturias, 2008). Muchas de estas investigaciones centran su interés en el patrón de consumo presentado, caracterizado por un rechazo del consumo de alcohol y tabaco (fundamentalmente motivado por prohibiciones ligadas a la religión), apareciendo como sustancias más consumidas los disolventes/inhalantes -dado su bajo precio y fácil adquisición-, seguidos del hachís -su consumo goza de cierta permisividad en Marruecos, por tratarse de país productor de dicha droga- (Markez y Pastor, 2009, 2010). Entre las motivaciones para dicho consumo abusivo, estos menores verbalizan la evasión de los problemas y de la realidad, la desinhibición para "quitar la sensación de miedo", así como la facilitación de la transgresión para cometer actos delictivos (Arbex y Jiménez, 2004; Pilatti, Brussino y Godoy, 2013).

De este modo, la literatura científica referente a la salud mental de estos menores se ve reducida a su consumo de sustancias, siendo inexistentes los estudios en torno al perfil psicológico de los mismos. Bien es cierto que hay multitud de programas de intervención dirigidos a menores infractores (extranjeros o no) que señalan la importancia de intervenir en aspectos como la autoestima, empatía, autocontrol, habilidades de comunicación interpersonal, impulsividad, locus de control externo, etc. (López, Carpintero, Campo, Lázaro y Soriano, 2011; Redondo, Martínez y Pueyo, 2011).

Las limitaciones de los recursos residenciales destinados a estos menores, los retrasos y dificultades en los procedimientos que facilitarían su integración, las dificultades de escolarización y respuesta educativa, sus escasas posibilidades de emancipación, las insuficientes prestaciones sanitarias a la 
hora de garantizar el derecho a la salud, y la imagen social transmitida de este colectivo como la de un grupo homogéneo, y especialmente conflictivo, sitúa a los MENAs en una situación de especial vulnerabilidad (Institución del Ararteko, 2011; Lenta, 2013). Estudios realizados con MENAs infractores (Capdevila y Ferrer, 2003) ofrecen un perfil diferenciado: varones, procedentes mayoritariamente de países del Magreb, que no inician su actividad delictiva de forma inmediata a su llegada, infractores fundamentalmente contra el patrimonio y a los cuales se les impone mayoritariamente internamiento cautelar en centro educativo.

Frente a este perfil de menor extranjero, se sitúan aquellos procedentes de reagrupaciones familiares (los acompañados). Desde el punto de vista del menor, este proceso implica dos grandes retos: en un primer momento, la residencia en el país nativo sin sus figuras de referencia, bajo la supervisión de algún familiar; lo cual justificará la aparición de conductas disruptivas, como llamada de atención ante dichas ausencias y, a su vez, conllevará una pérdida de autoridad de los padres (Díaz, 2012; Fresneda, 2001); y posteriormente, tras la reagrupación familiar, un nuevo reto de adaptación al país de destino. Aunque la reagrupación familiar es un proceso documentado, son muy pocos los estudios centrados en estos menores desde la perspectiva de infractores (San Segundo, 2014).

Sea como fuere, nacional o extranjero, la LORPM prevé una serie de medidas susceptibles de ser impuestas a los menores que presenten un comportamiento infractor, atendiendo la elección de la medida no solo a la prueba y valoración jurídica de los hechos, sino especialmente a la edad, las circunstancias familiares y sociales, la personalidad y el interés del menor (Art. 7.3 LORPM). Por su parte, Salvador (2013) advierte una sobrerrepresentación de los menores extranjeros entre los receptores de las medidas más severas, encontrando una posible justificación en el intento de suplir la ausencia de un entorno familiar estable en el que el joven pueda reinsertarse.

Dada la escasez de investigaciones acerca de menores infractores que distingan entre ambos perfiles de menores extranjeros, el objetivo del estudio es establecer un perfil diferencial de los menores extranjeros acompañados y no acompañados a nivel sociodemográfico, problemática psicológica y delictiva. La hipótesis de partida es que los menores extranjeros no pueden ser tratados como grupo homogéneo.

\section{Muestra}

\section{Método}

La población objeto de estudio es la totalidad de menores infractores del Principado de Asturias (España), en el año 2012. De esta población inicial de 269 menores infractores, se han seleccionado los menores extranjeros, entendiendo como tales aquellos que no han nacido en territorio español $\mathrm{y}$ han vivido, por tanto, un proceso migratorio. De cara a la selección de la muestra, se han utilizado como criterios de inclusión la imposición de una medida judicial (incluyéndose aquellos casos en los que se haya suspendido la ejecución del fallo) y el ser extranjero, suponiendo criterios de exclusión las resoluciones absolutorias, sobreseimientos, soluciones extrajudiciales y el tener nacionalidad española. Así, la muestra ha quedado conformada por un total de 90 menores (el $33.46 \%$ del total de menores infractores en Asturias durante el año 2012). Teniendo en cuenta las cifras aportadas anteriormente referentes a la población de menores en edad de responsabilidad penal en Asturias en el año 2012, se advierte una sobrerrepresentación de este colectivo dentro del Sistema de Justicia Juvenil (el 2.13\% 
de extranjeros presenta un comportamiento infractor frente a solo el $0.53 \%$ de los españoles).

De cara a responder a los objetivos planteados, se han matizado ciertos aspectos respecto del proceso migratorio que sufren dichos menores, distinguiéndose dos grupos en función de su modo de llegada a España:

No acompañados. Los MENAs, aquellos que llegan a España completamente solos, con/sin el consentimiento y conocimiento previo de su familia, siendo el medio más habitual de llegada en los bajos de un camión/autobús o en patera $(n=29$; correspondiente al 32\%).

$\sim$ Acompañados. Incluye mayoritariamente a los menores que llegan a España tras un proceso de reagrupación familiar $(n=61$; equivalente al $67.78 \%$ ). Contiene dos casos cuyo proceso se inicia tras su inclusión en un programa vacacional para niños saharauis y también dos casos de adopción (2.22\%, respectivamente); estos tienen cabida en este apartado en la medida en que reflejan una situación cercana a la reagrupación familiar, dado que el menor es separado de su familia de origen, pero en ningún momento reside solo en territorio español.

\section{Procedimiento}

Tras haber sido autorizado el acceso y la recogida de los datos por la Fiscalía de Menores del Principado de Asturias, según lo dispuesto en la Ley Orgánica 15/1999, de 13 de diciembre, de Protección de datos de Carácter Personal, se ha procedido a la extracción de los mismos; se utilizan como fuentes de información las resoluciones judiciales, los informes del equipo técnico y de ejecución de medida. Ello se ha realizado a través de la Ficha Abreviada de Informes de Ejecución (Rodríguez y Becedóniz,
2007), que recoge información personal, familiar y escolar de los menores, al mismo tiempo que incluye datos respecto de la salud mental, actividad infractora cometida, consumo de drogas, etc. Las variables utilizadas en el estudio han sido:

Variables sociodemográficas. Incluyen el género, la nacionalidad y la edad. A la hora de analizar esta última, es necesario especificar el momento en el cual reviste interés conocer la edad del menor. Al respecto, se han considerado como edades relevantes la de llegada a España y la del primer delito no sancionado, lo cual permite conocer el tiempo que tarda en cometer su primera infracción en territorio español.

Variables de problemática psicológica. Este apartado incluye el consumo de sustancias realizado por el menor: tipo de sustancia (legal/ilegal) e intensidad del consumo (uso/abuso). No obstante, también se han recogido indicadores psicológicos detectados por los profesionales del equipo técnico como relevantes en la socialización de este colectivo, los cuales aparecen recogidos en el correspondiente informe realizado y permitirán identificar las características más habituales de estos dos perfiles de menores extranjeros. Asimismo, dado que existe la posibilidad de que el Juez de Menores, además de imponer una medida judicial oriente unos objetivos de intervención de cara al cumplimiento de la medida -sobre la base de la información aportada por el equipo técnico-, parece relevante que estos sean incluidos como indicadores de la salud psicológica de estos menores ya que reflejan, en cierta medida, carencias en las cuales sería recomendable intervenir durante el cumplimiento de la medida.

Variables delictivas. La variable reincidencia es analizada teniendo en cuenta el número de infracciones cometidas por cada 
menor a lo largo de toda su carrera delictiva (lo cual no necesariamente coincide con el número de expedientes judiciales sancionados ni con el número de medidas impues$\left.\operatorname{tas}^{1}\right)$. Se identifica el tipo de infracciones cometidas a lo largo de su trayectoria delictiva, utilizando la categorización establecida por el Código Penal español (Ley orgánica 10/1995, de 23 de noviembre), así como diferenciando en función de si se trata de una infracción contra las personas (dentro de las cuales se incluyen las lesiones y aquellas contra la autoridad, la libertad, la libertad sexual, el honor y las relaciones familiares) o no (tales como infracciones contra el patrimonio, la Administración de Justicia y la seguridad colectiva). Asimismo se recoge la primera infracción cometida, momento relevante desde un punto de vista criminológico por marcar el inicio de la carrera delictiva del menor (Bringas, Rodríguez, Gutiérrez y Pérez-Sánchez, 2010; Bringas, Rodríguez, Moral, Pérez y Ovejero, 2012; Rodríguez et al., 2011), a la vez que se analizan las medidas judiciales impuestas - privativas o no de libertad $^{2}$ - a lo largo de toda su trayectoria delictiva -se destaca la medida judicial impuesta ante la primera infracción cometida, con el objetivo de comprobar la relación entre infracción cometida y medida judicial impuesta.

\section{Análisis de datos}

Los datos han sido procesados mediante el paquete estadístico SPSS 21.0 para Windows. En un primer momento se han efec-

\footnotetext{
${ }^{1}$ Es posible que en un mismo expediente judicial sea sancionada más de una infracción (Art. 11.1. LORPM), así como que una sola infracción sea sancionada con dos medidas diferentes (Art. 7.4. LORPM).

2 Se consideran medidas privativas de libertad, el internamiento en todas sus modalidades, así como la permanencia en fin de semana (tanto en un centro como en el domicilio); el resto de medidas, por tanto, son consideradas como no privativas de libertad.
}

tuado análisis descriptivos de las variables de estudio. Posteriormente, y con el objetivo de comparar los dos perfiles de menores infractores (acompañados y no acompañados), dado que se trata de una variable dicotómica, se utilizó el estadístico $X^{2}$ para las variables nominales, presentando el Odds Ratio como medida de la asociación entre dichas variables (Schiaffino et al., 2003). Para identificar las realidades diferenciales en las variables cuantitativas recogidas se realizaron Pruebas $U$ de Mann-Withney, dado que en ningún caso se cumplieron los supuestos paramétricos, siendo calculado el tamaño del efecto mediante el estadístico $d$ de Cohen (Cohen, 1988).

\section{Resultados}

\section{Perfil sociodemográfico}

Género. La mayoría de los menores son varones $(90 \%)$; concretamente, la totalidad de los MENAs $(n=29)$ y el $85.2 \%(n=52)$ de los menores acompañados.

Edad. Los resultados obtenidos constatan, con un tamaño del efecto alto, diferencias estadísticamente significativas entre ambos grupos en lo que respecta a la edad de llegada a España: los menores acompañados llegan significativamente más temprano que los MENAs (aproximadamente cuatro años antes (los acompañados lo hacen a los 9.8 años, mientras los MENAs llegan en torno a los 14 años) ( $U$ de MannWhitney $=252000 ; p=.000 ; d$ de Cohen $=$ 1092); sin embargo, ambos cometen su primera infracción en torno a la misma edad (los 15 años y medio) ( $U$ de Mann-Whitney $=780000 ; p=.367$ ). Así, esta realidad permite advertir diferencias (con un tamaño del efecto alto) en el tiempo transcurrido desde su llegada a España y la realización de su primera conducta infractora: mientras los MENAs tardan aproximadamente un año y medio en delinquir, los acompañados llevan 
casi seis años en España sin cometer infracción alguna ( $U$ de Mann-Whitney = 178500; $p=.000 ; d$ de Cohen $=0.79$ ).

Origen. Los MENAs proceden fundamentalmente de Marruecos (solo hay un caso cuyo país de origen es Portugal), siendo la procedencia de los menores acompañados más heterogénea: el $64 \%(n=39)$ procede de países latinoamericanos, mayoritariamente de Ecuador ( $n=12$; equivalente al 20\%), Brasil ( $n=10$; correspondiente al $16 \%)$ y Colombia $(n=8$; alcanzando un $13 \%)$; el $25 \%(n=15)$, de países del este de Europa, principalmente de Rumanía ( $n=$ 12; que alcanzan al 20\%); el 10\%, de África (tres casos de Argelia y otros tres de Marruecos); y el caso restante de Portugal.

\section{Problemática psicológica}

Consumo de sustancias. La mayoría de los menores reconoce consumir algún tipo de sustancia, exceptuando el $10.3 \%$ $(n=3)$ de los MENAs y el $19.7 \%(n=12)$ de los menores acompañados. Sin embargo, lo realmente interesante no es conocer las tasas de consumo, sino analizar el tipo de sustancia consumida y la intensidad del consumo. Los resultados no revelan diferencias estadísticamente significativas respecto de la intensidad del consumo $\left(X^{2}\right.$ $(2)=1.658 ; p=.198)$, ni siquiera en función del tipo de sustancia consumida $\left(X^{2}\right.$ (2) para sustancias legales $=2.109 ; p=$ $.146 ; X^{2}$ (2) para sustancias ilegales = $1.534 ; p=.215)$. A nivel meramente descriptivo, se puede concluir que entre las drogas legales (tabaco y alcohol), los MENAs abusan en mayor medida del tabaco ( $n=3$; correspondiente al $10.3 \%)$ y los acompañados del alcohol $(n=10$; que equivale al $16.4 \%$ ); mientras que en el caso de las drogas ilegales, los MENAs consumen en mayor medida inhalantes ( $n$ $=21$; es decir, un 38\%) y los acompañados cannabis $(n=30$; que alcanzan un
16.4\%). En cambio, sí se encuentran diferencias estadísticamente significativas en lo que respecta al consumo (o no) de sustancias ilegales: el $82.8 \%(n=24)$ de los MENAs confiesa ser consumidor de cannabis, éxtasis, inhalantes o fármacos (ninguno de los menores afirma consumir cocaína, heroína o ketamina), mientras que este valor disminuye hasta el $49.2 \%$ $(n=30)$ para los menores acompañados $\left(X^{2}(2)=9.234 ; p=.002 ; O R=4.960 ; I C\right.$ $=1.674-14.700)$.

Por último, queda en evidencia que las cifras relativas a la derivación de estos menores a programas de tratamiento de deshabituación de sustancias (seis casos entre los menores acompañados $\mathrm{y}$ tres entre los MENAs) son irrisorias. Los recursos utilizados para este fin han sido el Programa Reciella de Proyecto Hombre y los Centros de Salud Mental del Principado de Asturias.

Indicadores psicológicos. En ambos grupos se señalan altos niveles de impulsividad, dificultades en el cumplimiento de normas, límites y horarios, dependencia grupal (gran necesidad de pertenencia al grupo, lo cual lleva a que estos menores sean altamente influenciables) y déficit en la resolución de conflictos. Sin embargo, mientras en los menores acompañados destaca su bajo nivel de autoestima ( $n=$ 18; equivalente al $29.5 \%$ ), su tendencia al aislamiento y la soledad ( $n=10$; que alcanza un $16.4 \%$ ), déficit en habilidad sociales y asertividad ( $n=8$; correspondiente a un $13.1 \%)$ y sintomatología depresiva ( $n$ = 9; es decir, un 14.8\%); los MENAs se caracterizan por ser irresponsables $(n=$ 14 ; esto es, un $48.3 \%)$, inmaduros ( $n=9$; equivalente al $31 \%)$, desconfiados $(n=8$; que corresponde a un $27.6 \%$ ), inestables emocionalmente ( $n=13$; es decir, un $44.8 \%$ ), tener escasa competencia de autocontrol ( $n=11$; un $37.9 \%$ de los casos) 
y dificultades en los hábitos de higiene y sanitarios, así como en las rutinas y hábitos de convivencia ( $n=3$; que se asocia con un $10.3 \%$ ). Los niveles de medicación recibida por estos menores son bajos (solamente dos casos de menores acompañados y otros tres de MENAs).

Objetivos de la medida judicial. En el caso de los menores acompañados, estos son especificados en el $46.8 \%$ del total de 111 sentencias impuestas $(n=$ 52), mientras que solo 13 de las 165 sentencias impuestas a los MENAs aportan esta información (7.9\%). Los principales objetivos planteados permiten concluir, según lo reflejado en la tabla 3, que mientras que en el caso de los MENAs la intervención se centra en la prevención del consumo de tóxicos, los objetivos planteados para los menores acompañados son mucho más concretos y están orientados principalmente hacia la reflexión y asunción de las consecuencias de sus actos -y más concretamente, del hecho delictivo- y hacia la integración, a través del ámbito formativo-laboral, mediante el refuerzo de habilidades necesarias en toda relación interpersonal (asertividad, habilidades sociales, resolución de conflictos, etc.). Estos datos sugieren de manera evidente que se trata de perfiles con necesidades diferentes: el consumo de sustancias versus mejora de las relaciones interpersonales.

\section{Perfil delictivo}

Reincidencia. Mientras que los menores acompañados han cometido a lo largo de su carrera delictiva 2.5 infracciones de media (siendo 10 el máximo de conductas infractoras cometidas por uno de estos menores), los MENAs rondan las ocho infracciones de media (llevando a cabo uno de los menores un total de 23 infracciones), lo cual se traduce en diferencias estadísticamente significativas entre ambos grupos y un tamaño del efecto alto $(U$ de Mann-Whitney $=409000 ; p$ $=.000 ; d$ de Cohen $=1.14)$. Estos resultados implican en términos de reincidencia que mientras la mayoría de los menores acompañados (60.7\%; esto es, $n=37)$ son primarios (han cometido una sola infracción), en el caso de los MENAs predominan los multirreincidentes, es decir, aquellos que han cometido un mínimo de tres infracciones $(65.5 \% ; n=19)\left(X^{2}(2)\right.$ $=17.586 ; p=.000)$.

Infracciones cometidas. Acorde con la clasificación delictiva propuesta por el Código Penal, se pueden observar diferencias estadísticamente significativas, entre los menores acompañados y los que no lo están, respecto de la comisión de infracciones contra el orden público -se incluyen infracciones contra agentes de la autoridad y relacionadas con la venta/tráfico de armas $-\left(X^{2}(2)=30.110 ; p=\right.$ $.000 ; O R=31.607 ; I C=6.469-154.420) \mathrm{y}$ lesiones $\left(X^{2}(2)=7.771 ; p=.005 ; O R=\right.$ $3.780 ; I C=1.446-9.882)$, siendo ambas cometidas predominantemente por los MENAs (en concreto por el $52 \%$ y $72 \%$ de ellos, respectivamente), frente al $3.3 \%$ y $41 \%$ de menores acompañados que cometen dichas infracciones.

Analizando las infracciones desde un punto de vista criminológico, en función de si el objeto de las mismas son otras personas o no, los resultados ofrecen diferencias estadísticamente significativas $\left(X^{2}(2)=7.370 ; p=.007 ; O R=3.961 ; I C\right.$ $=1.41-11.09)$, en tanto los MENAs cometen infracciones contra las personas en mayor medida que los acompañados $(79 \% ; n=23$, frente a $49 \% ; n=30$, respectivamente). 
Tabla 3

Objetivos planteados en la sentencia de cara al cumplimiento de la medida judicial

\begin{tabular}{lcc} 
& $\begin{array}{c}\text { Acompañados } \\
(n=52)\end{array}$ & $\begin{array}{c}\text { No acompañados } \\
(n=13)\end{array}$ \\
\hline Asunción de las consecuencias de sus actos & 12 & 0 \\
Apoyo y estructuración del funcionamiento del menor & 8 & 3 \\
Orientación formativo-laboral & 8 & 1 \\
Resolución de conflictos & 8 & 0 \\
Evitar reincidencia (prevención de & 7 & 0 \\
comportamientos delictivos) & & \\
Asertividad & 7 & 0 \\
Prevención del consumo de tóxicos & 6 & 4 \\
Mejora de habilidades sociales & 6 & 1 \\
Ocupación del tiempo libre & 5 & 0 \\
Apoyo e integración educativa, social y laboral & 4 & 3 \\
Mejora de las relaciones familiares & 3 & 0 \\
Autoestima & 2 & 0 \\
Control de impulsos & 2 & 0 \\
Asunción de responsabilidades & 2 & 0 \\
Normalización de horarios & 2 & 0 \\
Déficits personales & 2 & 0 \\
Alfabetización & 0 & 2 \\
\hline
\end{tabular}

Nota: En una misma sentencia pueden plantearse varios objetivos.

Primera infracción cometida. La primera infracción que por lo general cometen ambos perfiles de menores infractores extranjeros suele ser contra el patrimonio $\left(X^{2}(2)=.230 ; p=.631\right)$, seguida de lesiones $\left(X^{2}(2)=.248 ; p=.618\right)$. No se identifican diferencias estadísticamente significativas entre los menores acompañados y los MENAs a la hora de cometer su primera infracción, incluso analizando la gravedad de las infracciones, tanto contra el patrimonio $\left(X^{2}(2)=1092 ; p=.296\right)$ -considerando el robo con violencia/intimidación y el robo con fuerza en vivienda de mayor gravedad que el resto (hurto, apropiación indebida, daños y robo con fuerza)-, como de lesiones, siendo cometidas las lesiones más graves (aquellas en las que son utilizadas armas) prácticamente en la misma medida por MENAs $(n=1)$ que por los menores acompañados $(n=2)$.

Medida judicial impuesta. Es necesario resaltar que en ningún caso se impusieron las medidas de internamiento en régimen abierto, internamiento terapéutico cerrado o abierto, tratamiento ambula- 
torio, amonestación, privación de permisos e inhabilitación absoluta. Los resultados, a su vez, indican que mientras las medidas judiciales impuestas con mayor frecuencia a los MENAs son el internamiento semiabierto (72\%), seguido de la permanencia de fin de semana en un centro $(52 \%)$ y las prestaciones en beneficio de la comunidad $(34.5 \%)$, la realidad es bien distinta respecto de los menores acompañados. A estos últimos, en la mayoría de los casos se les impone tareas socioeducativas $(47.5 \%)$, prestaciones en beneficio de la comunidad (41\%) y libertad vigilada (24.6\%). En este sentido, se observan diferencias estadísticamente significativas entre ambos grupos en lo que respecta a la imposición de la medida de internamiento en régimen semiabierto $\left(X^{2}(2)=27.318 ; p=.000 ; O R=\right.$ 13.388; $I C=4.64-38.62)$, permanencia de fin de semana en centro $\left(X^{2}(2)=\right.$ $26.914 ; p=.000 ; O R=20.714 ; I C=$ 5.26-81.53) -ambas impuestas predominantemente sobre MENAs-, frente a las tareas socioeducativas cumplidas mayoritariamente por menores acompañados $\left(X^{2}\right.$ (2) $=5.963 ; p=.015 ; O R=.288 ; I C=$ .103-.806). Se obtienen resultados en la misma línea en función de si la medida impuesta es o no privativa de libertad: los MENAs cumplen medidas privativas de libertad con una frecuencia significativamente mayor que aquellos acompañados $\left(X^{2}(2)=22.928 ; p=.000 ; O R=10.551\right.$; $I C=3.73-29.821)$, al contrario de lo que sucede con las medidas no privativas de libertad, impuestas con una frecuencia significativamente mayor a los menores acompañados $\left(X^{2}(2)=10.126 ; p=.001\right.$; $O R=.179 ; I C=.06-.55)$.

Primera medida judicial impuesta. Mientras las primeras medidas que suelen cumplir los menores acompañados son tareas socioeducativas $(n=26$; un $42.6 \%$ ), prestaciones en beneficio de la comunidad ( $n=16$; el $26.2 \%)$ y libertad vigilada ( $n=9$; un $14.8 \%$ ); en el caso de los MENAs, las primeras medidas que les son impuestas son el internamiento $(n=$ 8; esto es, el $27.6 \%$ ), y la permanencia de fin de semana $(n=6$; que alcanza a un $20.7 \%$ ). De esta manera, se observan diferencias significativas en el sentido de que las medidas privativas de libertad son impuestas en mayor medida a los MENAs que a los acompañados $\left(X^{2}(2)=14.031\right.$; $p=.000 ; O R=6.625 ; I C=2.32-18.92)$.

\section{Discusión y conclusiones}

Los datos obtenidos en nuestro estudio confirman, en primer lugar, la hipótesis de sobrerrepresentación de los menores extranjeros dentro del Sistema de Justicia Juvenil. La pregunta que se plantea entonces es la siguiente: ¿Todos los menores extranjeros reinciden en mayor medida? o, por el contrario, ¿no se trata de un grupo homogéneo, existiendo perfiles diferenciales dentro de dicho colectivo?

Los resultados en este contexto revelan similitudes entre los menores infractores extranjeros en lo que respecta a la edad de la primera infracción cometida -en torno a los 15 años y medio, de acuerdo con Bringas et al. (2010, 2012) y Rodríguez et al. (2011) - y la infracción con la que inician su carrera delictiva $(65 \%$ son conductas infractoras contra el patrimonio, seguidas de un $18 \%$ de lesiones), siendo también análogo el patrón de consumo de sustancias legales (26\% de abuso, fundamentalmente de alcohol) y las cifras de derivación a tratamientos de deshabituación insignificantes (alrededor del 10\%).

Sin embargo, las diferencias localizadas entre los menores acompañados y los no acompañados son múltiples. La primera de ellas hace referencia al género: aunque el $90 \%$ de la muestra de menores extran- 
jeros son varones -resultado coherente con los estudios anteriores de Bermejo et al. (2006), Capdevila y Ferrer (2003) y Markez y Pastor (2009)-, ha de destacarse que todas las mujeres infractoras extranjeras pertenecen al grupo de menores acompañados. Asimismo, los menores acompañados llegan a una edad significativamente más temprana a España y, por lo tanto, tardan significativamente más tiempo en cometer su primera infracción en territorio español. No obstante, se confirma el resultado obtenido por Capdevilla y Ferrer (2003) referente a que los MENAs no emigran con la intención de delinquir, ya que lejos de iniciar su carrera delictiva de forma inmediata a su llegada, tardan en delinquir una media de un año y medio.

En lo referente a la nacionalidad, mientras los MENAs proceden mayoritariamente de Marruecos -de acuerdo con Bermejo et al. (2006), Capdevila y Ferrer (2003), Procuradora General del Principado de Asturias (2008) y Markez y Pastor (2009)-, los acompañados lo hacen de países latinoamericanos. Esto conlleva diferencias culturales importantes entre ambos grupos: mientras los MENAs difieren tanto en idioma como en costumbres religiosas con la cultura española, en el caso de los menores acompañados el choque cultural es menos acusado. A nivel de problemática psicológica se trata de menores con necesidades muy diferentes: mientras los menores acompañados presentan problemas de adaptación y relaciones interpersonales, los MENAs manifiestan graves problemas de consumo; sin embargo, no son derivados en mayor medida a tratamientos de deshabituación, a la vez que sus objetivos de intervención planteados en la sentencia se dejan sin especificar en un $92 \%$ de los casos; ello es coherente con la literatura científica al respecto, según la cual los MENAs con- sumen sustancias ilegales en tasas significativamente mayores a las presentadas por los menores acompañados, confirmándose su problemática con el abuso de inhalantes/disolventes puesta de manifiesto por Markez y Pastor $(2009,2010)$.

Por último, resulta muy significativo el perfil diferencial a nivel delictivo presentado por estos menores. Las diferencias son claras cuando se analizan sus carreras delictivas: los MENAs reinciden significativamente más que los acompañados (cometen de media seis infracciones más) y las infracciones que cometen son más graves, en el sentido de que delinquen en mayor medida contra las personas (encontrándose diferencias estadísticamente significativas en infracciones contra el orden público, fundamentalmente contra agentes de la autoridad, y lesiones). De este modo, resulta coherente que a estos menores se les impongan medidas privativas de libertad (concretamente, internamiento en régimen semiabierto y permanencia de fin de semana en un centro) en mayor medida que a los acompañados, tanto por tratarse de reincidentes como por cometer infracciones de mayor gravedad. Pero ¿qué sucede si se elimina la variable reincidencia analizando la infracción con la que inician su carrera delictiva estos menores? En este caso, los resultados indican que cometen el mismo tipo de infracciones (contra el patrimonio y lesiones) y de la misma gravedad, por lo que sería de esperar que no apareciesen diferencias tampoco en lo que respecta a la medida judicial impuesta asociada a esa primera infracción. Sin embargo, los resultados constatan lo contrario, es decir, mientras el $87 \%$ de los menores acompañados cumple medidas no privativas de libertad (como tareas socioeducativas, prestaciones en beneficio de la comunidad y libertad vigilada), al 50\% de los MENAs se le impone medidas privativas 
de libertad (internamiento y permanencias de fin de semana). Entonces, si no es debido al nivel de reincidencia ni gravedad de la infracción, ¿a qué responde una mayor punitividad en el caso de los MENAs? Se confirma así la sobrerrepresentación entre los receptores de las medidas más severas, ya encontrada por Salvador (2013), pero en este caso no de los menores extranjeros como grupo homogéneo, sino concretamente de los MENAs.

Hay que reconocer que esta respuesta se ajusta a las indicaciones recogidas en la LORPM referentes a la elección de la medida en función del interés superior del menor, y no de la valoración jurídica de la infracción cometida. Aquí surge el debate acerca de cómo se concreta el concepto del interés superior del menor (Díaz, 2012; Lenta, 2013). En tanto que Salvador (2013) plantea la posibilidad de que el internamiento trate de suplir la ausencia de un entorno familiar estable, pero ¿puede una medida de carácter punitivo y restrictiva de la libertad del menor sustituir un entorno familiar estable?, ¿no sería mejor reforzar la intervención realizada desde el Sistema de Protección, o bien, orientar la intervención con estos menores hacia tratamientos de deshabituación, dado su grave problemática de adicción? En este momento, cobraría sentido la fase de inadaptación subjetiva (RodríguezDíaz y Paíno, 1994; Valverde, 2002) según la cual es la institucionalización del menor la que favorece su marginación (absolutamente todos los menores a los que se les impuso una medida privativa de libertad ante su primera infracción cometida reinciden, y el $77 \%$ de ellos son multirreincidentes). Otras variables no incluidas en este estudio, como podrían ser las tasas de fuga de los centros de protección por parte de los MENAs y el nivel de absentismo escolar podrían explicar los argumentos que subyacen en la decisión de imponer a estos menores medidas más punitivas, y su análisis podría contribuir a un conocimiento más certero de sus implicaciones de cara a la intervención desde los Sistemas de Protección y de Justicia.

Las limitaciones presentadas, así como aquellas asociadas a la recogida de datos a través de expedientes judiciales sin contrastación con evaluaciones directas de los menores abren la puerta a futuras investigaciones, a través de las cuales profundizar, sobre todo, en el perfil psicológico de dichos menores.

\section{Referencias}

Agencia Estatal Boletín Oficial del Estado, BOE. (2006). Ley Orgánica 8/2006 de 4 de diciembre, por la que se modifica la Ley Orgánica 5/2000, de 12 de enero, reguladora de la responsabilidad penal de los menores. Recuperado de http://www.boe.es/boe/dias/2006/12/05/pdfs/A42700-42712.pdf

Agencia Estatal Boletín Oficial del Estado, BOE. (2011). Real Decreto 557/2011 de 20 de abril, por el que se aprueba el Reglamento de la Ley Orgánica 4/2000, sobre derechos y libertades de los extranjeros en España y su integración social, tras su reforma por la Ley Orgánica 2/2009. Recuperado de http://www.boe.es/boe/dias/2011/04/30/pdfs/BOE-A-2011-7703.pdf 
Arbex, C. y Jiménez, A. (2004). Menores inmigrantes y consumo de drogas: un estudio cualitativo. Madrid: Ministerio del Interior y Plan Nacional de Drogas. Recuperado de http://jcpintoes.en.eresmas.com/drogas.pdf

Bermejo, F., Cario, R., Dünkel, F., Patanè, V. y Zapulla, A. (2006). Análisis criminológico del menor inmigrante infractor en el contexto europeo. En C. San Juan y J. De la Cuesta (Eds.), Menores extranjeros infractores en la Unión Europea. Teorías, perfiles y propuestas de intervención (pp. 15-56). Bilbao: Universidad del País Vasco, Servicio Editorial.

Bringas, C., Rodríguez, F. J., Gutiérrez, E. y Pérez-Sánchez, B. (2010). Socialización e historia penitenciaria. Revista Iberoamericana de Psicología y Salud, 1(1), 101-116. Recuperado de http://www.redalyc.org/articulo.oa?id=245116411008

Bringas, C., Rodríguez, F. J., Moral, M. V., Pérez, B. y Ovejero, A. (2012). Comportamiento delictivo reincidente. Análisis diferencial de la variable edad. Revista Interamericana de Psicología/Interamerican Journal of Psychology, 46(3), 365-374. Recuperado de http://www.redalyc.org/pdf/284/28425871005.pdf

Capdevila, M. y Ferrer, M. (2003). Los menores extranjeros indocumentados no acompañados (MEINA). Generalitat de Catalunya: Centre d'Estudis Jurídics i Formació especialitzada.

Cohen, J. (1988). Statistical power analysis for the behavioral sciences ( $2^{\mathrm{a}}$ ed.). New Jersey: Erlbaum.

Díaz, D. A. (2012). Servicio en beneficio de la comunidad: una aproximación cuantitativa a la justicia juvenil restaurativa en Chile. Revista de Psicología, 21(2), 83-107. http://dx.doi.org/10.5354/0719-0581.2012.25838

Estrada, C., Herrero, J. y Rodríguez Díaz, F. J. (2014). Rol de apoyo social en la reincorporación de penados. Un estudio del sistema de postpenitenciario de Jalisco (México). Universitas Psichologica, 13(3), 15-28. Recuperado de

http://revistas.javeriana.edu.co/index.php/revPsycho/article/download/2755/8729

Fresneda, J. (2001). Redefinición de las relaciones familiares en el proceso migratorio ecuatoriano a España. Migraciones Internacionales, 1(1), 135-144. Recuperado de http://www.colef.mx/migracionesinternacionales/revistas/MI01/n01-135-144.pdf

Garrido, V., Stangeland, P. y Redondo, S. (2006). Principios de criminología (3 ${ }^{\mathrm{a}}$ ed.). Valencia: Tirant lo Blanch.

Herrero, J., Rodríguez Díaz, F. J. y Musitu, G. (2014). Comparing intergroup contact effects on blatant and subtle prejudice in adolescents: A multivariate multilevel model. Psicothema, 26(1), 33-38.

http://www.dx.doi.org/10.7334/psicothema2013.163

Hirschi, T. (1969). Causes of delinquency. Berkeley: University of California Press.

Institución del Ararteko (2011). Infancias vulnerables: informe extraordinario de la Institución del Ararteko al Parlamento Vasco. Vitoria-Gasteiz: Ararteko. Recuperado de http://bibliotecadigital.org/jspui/handle/001/256 
Instituto Nacional de Estadística, INE. (2013). Estadística de condenados: menores. Recuperado de

http://www.ine.es/jaxi/menu.do?type=pcaxis\&path=/t18/p467\&file=inebase

Leiva, L., Pineda, M. y Encina, Y. (2013). Autoestima y apoyo social como predictores de la resiliencia en un grupo de adolescentes en vulnerabilidad social. Revista de Psicología, 22(2), 111-123.

http://dx.doi.org/10.5354/0719-0581.2013.30859

Lenta, M. M. (2013). Niños y niñas en situación de calle: territorios, vínculos y políticas sociales. Revista de Psicología, 22(2), 29-41.

http://dx.doi.org/10.5354/0719-0581.2013.30851

López, F., Carpintero, E., Campo, A., Lázaro, S. y Soriano, S. (2011). Programa menores infractores: intervención educativa y terapéutica. Madrid: Pirámide.

Markez, I. y Pastor, F. (2009). Drogodependencia en menores extranjeros no acompañados (MENA): su derecho a una educación y salud de calidad. Vitoria-Gasteiz: Servicio Central de Publicaciones del Gobierno Vasco. Recuperado de http:/is.gd/g3iWP7

Markez, I. y Pastor, F. (2010). Menores extranjeros no acompañados (MENA), un colectivo especialmente vulnerable ante las drogas. Zerbitzuan, 48, 71-86. Recuperado de http://is.gd/YcnQms

Montero, H. T. (2014). La criminalidad juvenil en España (2007-2012). Revista Criminalidad, 56(2), 247-261. Recuperado de

http://www.policia.gov.co/imagenes_ponal/dijin/revista_criminalidad/vol56_2/08.pdf

Moreno, G. (2012). Actitudes y opinión sobre los menores extranjeros no acompañados en la comunidad autónoma del País Vasco. Migraciones, 31, 43-68. Recuperado de http://revistas.upcomillas.es/index.php/revistamigraciones/article/view/921/779

Musitu, G., Moreno, D. y Murgui, S. (2007). Adolescentes infractores: la perspectiva psicosocial. En F. J. Rodríguez-Díaz y C. Becedóniz (Coords.), El menor infractor: posicionamientos y realidades (pp. 13-36). Oviedo: Consejería de Justicia, Seguridad Pública y Relaciones Exteriores.

Paíno, S. (2008). Factores de riesgo y de protección en la infancia y la adolescencia. En F. J. Rodríguez, C. Bringas, F. Fariña, R. Arce y A. Bernardo (Eds.), Psicología jurídica. Entorno judicial y delincuencia (pp. 151-172). Oviedo: Ediciones de la Universidad de Oviedo.

Pilatti, A., Brussino, S. A. y Godoy, J. C. (2013). Factores que influyen en el consumo de alcohol de adolescentes argentinos: un path análisis prospectivo. Revista de Psicología, 22(1), 22-36.

http://dx.doi.org/10.5354/0719-0581.2013.27716

Procuradora General del Principado de Asturias. (2008). La protección de los menores extranjeros no acompañados en Asturias. Recuperado de http://is.gd/FNf7mI 
Redondo, S. (2008). Individuos, sociedades y oportunidades en la explicación y prevención del delito: modelo del Triple Riesgo Delictivo (TRD). Revista Española de Investigación Criminológica, 7(6), 1-53. Recuperado de http://www.criminologia.net/pdf/reic/ano6-2008/a62008art7.pdf

Redondo, S., Martínez, A. y Pueyo, A. A. (2011). Factores de éxito asociados a los programas de intervención con menores infractores. Madrid: Ministerio de Sanidad, Política social e igualdad. Recuperado de http://www.observatoriodelainfancia.msssi.gob.es/productos/pdf/factoresDeExito.pdf

Rodríguez Díaz, F. J. y Becedóniz, C. Coord. (2007). El menor infractor. Posicionamientos y realidades. Oviedo: Gobierno del Principado de Asturias.

Rodríguez, F. J. y Paíno, S. G. (1994). Violencia y desviación social. Bases y análisis para la intervención. Psicothema, 6(2), 229-244.

Rodríguez Díaz, F. J., Bringas, C., Rodríguez-Franco, L., López-Cepero, J., Pérez, B., \& Estrada, C. (2011). Drug abuse and criminal family records in the criminal history of prisoners. The European Journal of Psychology Applied to Legal Context, 3(2), 89-105. Recuperado de http://is.gd/LXeh94

Rodríguez, F. J., Herrero, J., Ovejero, A., \& Torres, A. (2009). New expressions of racism among young people in Spain: An adaptation of the Meertens and Pettigrew (1992) prejudice scale. Adolescence, 44(176), 1033-1043. Recuperado de http://hdl.handle.net/10651/9859

Salvador, R. (2013). El menor inmigrante como infractor penal. Revista Internacional de Estudios Migratorios, 3(1), 1-24. Recuperado de http://www.riem.es/espanol/ContadorArticulo.php?idart=30

San Segundo, C. (2014). Perfil de menores infractores procedentes de reagrupaciones familiares (Trabajo Fin de Máster. Universidad de Oviedo, Asturias, España). Recuperado de http://hdl.handle.net/10651/27771

Schiaffino, A., Rodríguez, M., Pasarín, M., Regidor, E., Borrell, C. y Fernández, E. (2003). ¿Odds Ratio o razón de proporciones? Su utilización en estudios transversales. Gaceta Sanitaria, 17(1), 70-74. Recuperado de http://scielo.isciii.es/pdf/gs/v21n4/notaeditorial1.pdf

Valdenegro, B. A. (2005). Factores psicosociales asociados a la delincuencia juvenil. Psykhe, 14(2), 33-42.

http://dx.doi.org/10.4067/S0718-22282005000200003

Valverde, J. (2002). Proceso de inadaptación social (4ª ed.). Madrid: Popular. 